\title{
On a System of Difference Equations of an Economic Model
}

\author{
M. F. Elettreby ${ }^{1,2}$ and H. El-Metwally ${ }^{2,3}$ \\ ${ }^{1}$ Department of Mathematics, Faculty of Science, King Khalid University, Abha 9004, Saudi Arabia \\ ${ }^{2}$ Department of Mathematics, Faculty of Science, Mansoura University, Mansoura 35516, Egypt \\ ${ }^{3}$ Department of Mathematics, Rabigh College of Science and Arts, King Abdulaziz University, P.O. Box 344, Rabigh 21911, Saudi Arabia
}

Correspondence should be addressed to H. El-Metwally; helmetwally@mans.edu.eg

Received 13 February 2013; Accepted 10 August 2013

Academic Editor: Garyfalos Papaschinopoulos

Copyright (c) 2013 M. F. Elettreby and H. El-Metwally. This is an open access article distributed under the Creative Commons Attribution License, which permits unrestricted use, distribution, and reproduction in any medium, provided the original work is properly cited.

We study some qualitative properties of the solutions of a system of difference equations, which describes an economic model. The study of the local stability of the equilibrium points is carried out. We give some important results of the invariant and the boundedness of the solutions to the considered system. The global convergence of the solutions is presented and investigated.

\section{Introduction}

The increasing study of realistic mathematical models is a reflection of their use in helping to understand the dynamic processes involved in areas such as population dynamics, biology, epidemiology, ecology, and economy. More realistic models should include some of the past states of these systems; that is, ideally, a real system should be modeled by difference equations with time delays. Most of these models are described by nonlinear delay difference equations; see, for example, [1-4]. The subject of the qualitative study of the nonlinear delay population models is very extensive, and the current research work tends to center around the relevant global dynamics of the considered systems of difference equations such as oscillation, boundedness of solutions, persistence, global stability of positive steady sates, permanence, and global existence of periodic solutions. See [5-18] and the references therein.

In this paper, we intend to cover some of these global aspects of the qualitative behavior of a system of a discrete model in the economy area, where we deal with the studying of some qualitative properties of solutions of the following system of difference equations:

$$
x_{n+1}=(1-\alpha) x_{n}+\beta x_{n}\left(1-x_{n}\right) e^{-\left(x_{n}+y_{n}\right)} \text {, }
$$

$$
\begin{array}{r}
y_{n+1}=(1-\alpha) y_{n}+\beta y_{n}\left(1-y_{n}\right) e^{-\left(x_{n}+y_{n}\right)}, \\
n=0,1, \ldots,
\end{array}
$$

where $\alpha$ and $\beta \in(0, \infty)$ with the initial conditions $x_{0}$ and $y_{0} \in$ $(0, \infty)$. We study the boundedness and the invariant of the solutions of system (1) and also investigate global convergence for the solutions of system (1).

System (1) is an important type of economic models which describes a discrete-time map generated by bounded rationally duopoly game with exponential demand function. See [19].

The following theorem was presented in [6], and it will be useful in the investigation of the global stability of system (1).

Theorem A. Consider the following system of difference equations:

$$
\begin{array}{r}
x_{n+1}=f\left(x_{n}, y_{n}\right), \\
y_{n+1}=g\left(x_{n}, y_{n}\right), \\
n=0,1, \ldots .
\end{array}
$$




\section{Suppose that}

(i) $f(x ; y)$ is nondecreasing in $x$ and is nonincreasing in $y$, and $g(x ; y)$ is nonincreasing in $x$ and is nondecreasing in $y$,

(ii) if $m_{1}=f\left(m_{1}, M_{2}\right) ; M_{1}=f\left(M_{1}, m_{2}\right) ; m_{2}=$ $g\left(M_{1}, m_{2}\right)$ and $M_{2}=f\left(m_{1}, M_{2}\right)$ imply $m_{1}=M_{1}$ and $m_{2}=M_{2}$.

Then, system (2) has a unique positive equilibrium point $(x, y)$, and every solution of system (2) converges to $(x, y)$.

The equilibrium points of system (1) are the solutions of the following system:

$$
\begin{aligned}
& \bar{x}=(1-\alpha) \bar{x}+\beta \bar{x}(1-\bar{x}) e^{-(\bar{x}+\bar{y})}, \\
& \bar{y}=(1-\alpha) \bar{y}+\beta \bar{y}(1-\bar{y}) e^{-(\bar{x}+\bar{y})}
\end{aligned}
$$

or

$$
\begin{aligned}
& \bar{x}=0, \quad \alpha=\beta(1-\bar{x}) e^{-(\bar{x}+\bar{y})}, \\
& \bar{y}=0, \quad \alpha=\beta(1-\bar{y}) e^{-(\bar{x}+\bar{y})} .
\end{aligned}
$$

El-Metwally and Elsadany [19] have shown that system (1) has the equilibrium points $(0,0),(x, 0),(0, x)$, and $(u, u)$ where $x$ and $u$ satisfy $\alpha=\beta(1-x) e^{-x}$ and $\alpha=\beta(1-u) e^{-2 u}$, respectively, and proved that

(i) the equilibrium point $(0,0)$ of system (1) is locally asymptotically stable if $\beta<\alpha<\beta+2$ and unstable if $\beta>\alpha$;

(ii) the equilibrium points $(x, 0)$ and $(0, x)$ of system (1) are unstable;

(iii) the equilibrium point $(u, u)$ of system (1) is stable if $\beta<$ $\left(4 \alpha^{2} /\left(\alpha-2+\sqrt{4-4 \alpha+9 \alpha^{2}}\right)\right) e^{(1 / 2 \alpha)\left(2+3 \alpha-\sqrt{4-4 \alpha+9 \alpha^{2}}\right)}$ and unstable if $\beta>\left(4 \alpha^{2} /(\alpha-2+\right.$ $\left.\left.\sqrt{4-4 \alpha+9 \alpha^{2}}\right)\right) e^{(1 / 2 \alpha)\left(2+3 \alpha-\sqrt{4-4 \alpha+9 \alpha^{2}}\right)}$.

\section{Boundedness and Invariant}

In this section, we concern ourselves with the boundedness character of solutions of system (1). Under appropriate conditions, we give some bounded results related to system (1).

Theorem 1. Assume that $\left\{\left(x_{n}, y_{n}\right)\right\}_{n=0}^{\infty}$ is a solution of system (1). Then,

(i) $x_{0}=y_{0}$ implies that $x_{n}=y_{n}$ for all $n=0,1, \ldots$,

(ii) $1-\alpha+\beta\left[1-\left(x_{0}+y_{0}\right)\right] e^{-\left(x_{0}+y_{0}\right)}=0$ implies that $x_{n}=y_{n}$ for all $n=0,1, \ldots$,

(iii) if $\alpha+\beta / e^{2}<1$, then $x_{n}>y_{n}\left(x_{n}<y_{n}\right)$ for all $n=$ $0,1, \ldots$ provided that $x_{0}>y_{0}\left(x_{0}<y_{0}\right)$.

Proof. It follows from (1) that

$$
x_{n+1}-y_{n+1}=\left(x_{n}-y_{n}\right)\left[1-\alpha+\beta\left(1-\left(x_{n}+y_{n}\right)\right) e^{-\left(x_{n}+y_{n}\right)}\right] \text {. }
$$

So Cases (i) and (ii) are immediately proved. Now set

$$
f(x)=1-\alpha+\beta(1-x) e^{-x} .
$$

Then,

$$
f^{\prime}(x)=\beta(x-2) e^{-x}
$$

Therefore, $f(2)$ is the absolute minimum of $f(x)$. That is,

$$
f(x) \geq f(2)=1-\alpha-\frac{\beta}{e^{2}}>0 .
$$

Note that (5) implies

$$
x_{n+1}-y_{n+1}=\left(x_{n}-y_{n}\right) f\left(x_{n}+y_{n}\right),
$$

and, hence, $\left(x_{n+1}-y_{n+1}\right)$ has the same sign of $\left(x_{n}-y_{n}\right)$ for all $n>0$. The proof is so complete.

Remark 2. Theorem 1 reduces system (1) into the following single difference equation:

$$
x_{n+1}=x_{n}\left[1-\alpha+\beta\left(1-x_{n}\right)\right] e^{-2 x_{n}} .
$$

Theorem 3. Assume that $\alpha+\beta / e^{2}<1$. Then, every solution $\left\{\left(x_{n}, y_{n}\right)\right\}_{n=0}^{\infty}$ of system (1) with $x_{0}>0$ and $y_{0}>0$ satisfies that $x_{n}>0$ and $y_{n}>0$ for all $n>0$.

Proof. Let $H(x, y)$ be a continuous function defined by

$$
H(x, y)=1-\alpha+\beta(1-x) e^{-(x+y)} .
$$

Then, system (1) can be rewritten in the form

$$
\begin{aligned}
& x_{n+1}=x_{n} H\left(x_{n}, y_{n}\right), \\
& y_{n+1}=y_{n} H\left(y_{n}, x_{n}\right) .
\end{aligned}
$$

Now assume that $\left\{\left(x_{n}, y_{n}\right)\right\}_{n=0}^{\infty}$ is a solution of system (1) with positive initial values. Then, it suffices to show that $H(x, y)$ is positive for all $x>0, y>0$. Observe that

$$
\begin{gathered}
\frac{\partial H(x, y)}{\partial x}=\beta(x-2) e^{-(x+y)}, \\
\frac{\partial H(x, y)}{\partial y}=-\beta(1-x) e^{-(x+y)} .
\end{gathered}
$$

Therefore, $H$ has no positive critical points. Let $a$ and $b$ be arbitrary positive numbers and consider the domain

$$
D=\{(x, y): 0 \leq x \leq a, 0 \leq y \leq b\} .
$$

Then,

$$
\begin{gathered}
H(0, y)=1-\alpha+\beta e^{-y}, \quad 0 \leq y \leq b, \\
H(x, 0)=1-\alpha+\beta(1-x) e^{-x}, \quad 0 \leq x \leq a, \\
H(x, b)=1-\alpha+\beta(1-x) e^{-(x+b)}, \quad 0 \leq x \leq a, \\
H(a, y)=1-\alpha+\beta(1-a) e^{-(a+y)}, \quad 0 \leq y \leq b .
\end{gathered}
$$


Using elementary differential calculus, we obtain that the absolute minimum of each one of the above functions is $1-\alpha-$ $\beta / e^{2}$. Therefore, $H(x, y) \geq 1-\alpha-\beta / e^{2}>0$ for all $(x, y) \in D$. Since $a$ and $b$ are arbitrary positive numbers, we can conclude that $H(x, y)>0$ for all $(x, y) \in(0, \infty)^{2}$.

Theorem 4. Assume that $\left\{\left(x_{n}, y_{n}\right)\right\}_{n=0}^{\infty}$ is a solution of system (1) with $\left(x_{n_{0}}, y_{n_{0}}\right) \in(0,1]^{2}$ for some $n_{0} \geq 0$. Assume also that one of the following statements is true.

(i) $\beta \leq e(1-\alpha)$.

(ii) $e(1-\alpha)<\beta \leq e$.

(iii) $(\sqrt{\beta}-1)^{2} \leq \alpha$.

Then, $\left(x_{n}, y_{n}\right) \in(0,1]^{2}$ for all $n \geq n_{0}$.

Proof. Let $n_{0} \geq 0$ be such that $x_{n_{0}} \in(0,1]$. It follows from system (1) that

$$
\begin{gathered}
x_{n_{0}+1} \leq(1-\alpha) x_{n_{0}}+\beta\left(1-x_{n_{0}}\right) x_{n_{0}} e^{-x_{n_{0}}}, \\
x_{n_{0}+1} \leq(1-\alpha) x_{n_{0}}+\beta\left(1-x_{n_{0}}\right) e^{-1} \\
=\left(1-\alpha-\frac{\beta}{e}\right) x_{n_{0}}+\frac{\beta}{e}, \\
x_{n_{0}+1} \leq(1-\alpha) x_{n_{0}}+\beta\left(1-x_{n_{0}}\right) x_{n_{0}} .
\end{gathered}
$$

Set $w(x)=(1-\alpha) x+\beta(1-x) x e^{-x}$ for $x \leq 1$. Then, it follows from (16) that $x_{n_{0}+1} \leq w\left(x_{n_{0}}\right)$. Also, we obtain that

$$
\begin{gathered}
w^{\prime}(x)=(1-\alpha)+\beta\left(x^{2}-3 x+1\right) e^{-x}, \\
w^{\prime \prime}(x)=\beta\left(-x^{2}+5 x-4\right) e^{-x}=-\beta(x-1)(x-4) e^{-x} \\
\leq 0 \quad \forall x \in(0,1] .
\end{gathered}
$$

Then, $w^{\prime}(x) \geq w^{\prime}(1)=1-\alpha-\beta / e$. If (i) holds, then $w^{\prime}(1) \geq$ 0 , and hence $w(x)$ is increasing on $(0,1]$. Therefore, $x_{n_{0}+1} \leq$ $w(1)<1$. If (ii) holds, then (17) yields $x_{n_{0}+1} \leq \beta / e<1$.

Now suppose that (iii) holds. In this case, it follows from (18) that $x_{n_{0}+1} \leq p\left(x_{n_{0}}\right)$, where $p(x)=(1-\alpha) x+\beta x(1-x)$ for all $x \in(0,1]$. It is not difficult to see that $p\left(x_{*}\right)$ is the absolute maximum of $p(x)$ on $(0,1]$ where $x_{*}=(1-\alpha+\beta) / 2 \beta$. According to (iii) and since $p\left(x_{*}\right)=(1-\alpha+\beta)^{2} / 4 \beta \leq$ $1, x_{n_{0}+1} \leq p\left(x_{*}\right) \leq 1$. That is in all cases we obtain that whenever $x_{n_{0}} \leq 1$ gives $x_{n_{0}+1} \leq 1$. So it is easy to prove by induction that $x_{n} \in(0,1]$ for all $n \geq 1$. The proof of $y_{n}$ is similar and so will be omitted. This completes the proof.

Theorem 5. For every solution $\left\{\left(x_{n}, y_{n}\right)\right\}_{n=0}^{\infty}$ of system (1), the following statements hold.

(i) $x_{n} \leq x_{n_{0}}(1-\alpha)^{n-n_{0}}+(\beta / e \alpha)\left(1-(1-\alpha)^{n-n_{0}}\right), n \geq n_{0} \geq 0$.

(ii) $y_{n} \leq y_{n_{0}}(1-\alpha)^{n-n_{0}}+(\beta / e \alpha)\left(1-(1-\alpha)^{n-n_{0}}\right), n \geq n_{0} \geq 0$.
Proof. We obtain, for $n_{0} \geq 0$, from (1) that

$$
\begin{aligned}
x_{n+1} & \leq(1-\alpha) x_{n}+\beta x_{n} e^{-x_{n}} \\
& \leq(1-\alpha) x_{n}+\frac{\beta}{e}, \quad \forall n \geq n_{0} .
\end{aligned}
$$

Then, it follows by Theorems 3 and 4 that Case (i) is true. The proof of Case (ii) is similar and so will be omitted.

The following corollaries are coming immediately from Theorem 5.

Corollary 6. Assume that $\left\{\left(x_{n}, y_{n}\right)\right\}_{n=0}^{\infty}$ is a positive solution of system (1) with $\left(x_{n_{0}}, y_{n_{0}}\right) \in(0, \beta / \alpha e]^{2}$ for $n_{0} \geq 0$. Then, $\left(x_{n}, y_{n}\right) \in(0, \beta / \alpha e]^{2}$ for all $n \geq n_{0}$.

Corollary 7. Every positive solution $\left\{\left(x_{n}, y_{n}\right)\right\}_{n=0}^{\infty}$ of system (1) is bounded. Moreover,

$$
\begin{aligned}
& \limsup _{n \rightarrow \infty} x_{n} \leq \frac{\beta}{\alpha e}, \\
& \limsup _{n \rightarrow \infty} y_{n} \leq \frac{\beta}{\alpha e} .
\end{aligned}
$$

In Theorem 4, we gave conditions under which every positive solution $\left\{\left(x_{n}, y_{n}\right)\right\}_{n=0}^{\infty}$ of system (1) to be in $(0,1]^{2}$ provided that $\left(x_{n_{0}}, y_{n_{0}}\right) \in(0,1]^{2}$. In the next result we show that every positive solution $\left\{\left(x_{n}, y_{n}\right)\right\}_{n=0}^{\infty}$ of system (1) eventually lies in $(0,1]^{2}$.

Theorem 8. Assume that $\left\{\left(x_{n}, y_{n}\right)\right\}_{n=0}^{\infty}$ is a positive solution of system (1), and assume that one of the following conditions is true.

(i) $\beta<\alpha e$.

(ii) $2+v^{2}+2 e^{\nu}-4 v-v e^{\nu}>0,1-\alpha+\beta e^{-v}\left[1-\nu\left(2 e^{-v}+\right.\right.$ $\left.1)+v^{2} e^{-v}\right]$ and $(1-\alpha) v+\beta v e^{-v}-\beta v^{2} e^{-2 v}<1$, where $\nu=\beta / \alpha e$.

Then, there exists $n_{0} \geq 0$ such that $\left(x_{n}, y_{n}\right) \in(0,1]^{2}$ for all $n \geq n_{0}$.

Proof. The proof of the theorem, when (i) holds, is followed by Corollary 7. Now consider that (ii) is true. Then, it follows from Corollary 7 that for every constant $\varepsilon>0$, there exists $n_{0} \geq 0$ such that $x_{n} \leq \beta / \alpha e+\varepsilon=\gamma, n \geq n_{0}$. Set $\delta=e^{-\gamma}$. Since $\delta \rightarrow e^{-v}$ when $\varepsilon \rightarrow 0$ and the inequalities in (ii) hold, depending on the continuity in $\nu$ of the left hand side of each inequality in (ii), one can choose $\varepsilon$ so small such that

$$
\begin{gathered}
2+\gamma^{2}+\frac{2-\gamma}{\delta}-4 \gamma \geq 0 \\
1-\alpha+\beta \delta\left[1-(2 \delta+1) \gamma+\delta \gamma^{2}\right] \geq 0 \\
(1-\alpha) \gamma+\beta \gamma \delta-\beta \gamma^{2} \delta^{2} \leq 1
\end{gathered}
$$


Now, we obtain from (1) that

$$
\begin{aligned}
x_{n+1} & =(1-\alpha) x_{n}+\beta x_{n} e^{-\left(x_{n}+y_{n}\right)}-\beta x_{n}^{2} e^{-\left(x_{n}+y_{n}\right)} \\
& \leq(1-\alpha) x_{n}+\beta x_{n} e^{-x_{n}}-\beta \delta x_{n}^{2} e^{-x_{n}} \\
& =K\left(x_{n}\right), \quad n \geq n_{0},
\end{aligned}
$$

where $K(x)=(1-\alpha) x+\beta e^{-x}\left(x-\delta x^{2}\right), x \leq \gamma$, and then

$$
\begin{aligned}
K^{\prime}(x) & =1-\alpha+\beta e^{-x}\left[\delta x^{2}-(2 \delta+1) x+1\right], \\
K^{\prime \prime}(x) & =-\beta e^{-x}\left[\delta x^{2}-(4 \delta+1) x+2(\delta+1)\right] .
\end{aligned}
$$

On the other hand, the equation

$$
\delta x^{2}-(4 \delta+1) x+2(\delta+1)=0,
$$

has the positive roots

$$
x_{1}=\frac{4 \delta+1+\sqrt{8 \delta^{2}+1}}{2 \delta}, \quad x_{2}=\frac{4 \delta+1-\sqrt{8 \delta^{2}+1}}{2 \delta} .
$$

Observe that $x_{2}=2+(1 / 2 \delta)-\sqrt{2+1 / 4 \delta^{2}} \geq \gamma$ if and only if $(2+(1 / 2 \delta)-\gamma)^{2} \geq 2+1 / 4 \delta^{2}$ which holds by (22). Therefore, $x_{1} \geq x_{2} \geq \gamma$. Consequently, $K^{\prime \prime}(x)<0$ for all $x \leq \gamma$ which yields by (23) that $K^{\prime}(x)>K^{\prime}(\gamma) \geq 0$. Using the increasing property of $K(x)$ on $(0, \gamma)$ and inequality (24), we see that $K(x) \leq K(\gamma) \leq 1$. Since $x_{n} \leq \gamma$, it follows that

$$
x_{n+1} \leq K\left(x_{n}\right) \leq K(\gamma) \leq 1 \quad \forall n \geq n_{0} .
$$

This completes the proof.

Theorem 9. Assume that $\left\{\left(x_{n}, y_{n}\right)\right\}_{n=0}^{\infty}$ is a positive solution of system (1). If either

$$
(1-\alpha+\beta)^{2}<4 \beta e^{-2 v}
$$

or

$$
\frac{\beta e^{\nu}}{4}+\beta(1-\alpha)<1
$$

where $v=\beta / \alpha$, then there exists $n_{0} \geq 0$ such that $\left(x_{n}, y_{n}\right) \in$ $(0,1]^{2}$ for all $n \geq n_{0}$.

Proof. Assume that $\gamma, \delta$, and the function $K\left(x_{n}\right)$ are defined as in the previous proof. Then,

$$
K\left(x_{n}\right)=(1-\alpha+\beta) x_{n}-\beta x_{n}^{2} \delta^{2}=\bar{K}\left(x_{n}\right),
$$

where $\bar{K}(x)=(1-\alpha+\beta) x-\beta x^{2} \delta^{2}, x \leq \gamma$. Thus,

$$
\overline{K^{\prime}}(x)=1-\alpha+\beta-2 \beta x \delta^{2} \text {. }
$$

Hence, $\bar{K}(x)$ attains its maximum value at $x=(1-\alpha+$ $\beta) / 2 \beta \delta^{2}$; that is,

$$
\bar{K}(x) \leq \bar{K}\left(\frac{1-\alpha+\beta}{2 \beta \delta^{2}}\right)=\frac{(1-\alpha+\beta)^{2}}{4 \beta \delta^{2}} .
$$

Also,

$$
\begin{aligned}
K\left(x_{n}\right) & =-\beta \delta e^{-x_{n}}\left(x_{n}-\frac{1}{2 \delta}\right)^{2}+\frac{\beta e^{-x_{n}}}{4 \delta}+\beta(1-\alpha) \\
& <\frac{\beta}{4 \delta}+\beta(1-\alpha), \quad n \geq n_{0} .
\end{aligned}
$$

Similar to the proof of Theorem 8 , we can choose $\varepsilon$ so small such that our assumptions imply that

$$
\frac{(1-\alpha+\beta)^{2}}{2 \beta \delta^{2}} \leq 1, \quad \frac{\beta}{4 \delta}+\beta(1-\alpha) \leq 1 .
$$

Therefore, we have either

$$
x_{n+1} \leq \bar{K}\left(x_{n}\right) \leq \frac{(1-\alpha+\beta)^{2}}{2 \beta \delta^{2}} \leq 1, \quad n \geq n_{0}
$$

or

$$
x_{n+1} \leq K\left(x_{n}\right) \leq \frac{\beta}{4 \delta}+\beta(1-\alpha) \leq 1, \quad n \geq n_{0},
$$

which is our desired conclusion for $x_{n}$. Similarly, one can accomplish the same conclusion for $y_{n}$. The proof is so complete.

\section{Global Stability Analysis}

In this section, we are interested in establishing conditions under which the equilibrium points of system (1) are to be the attractors of the solutions of system (1).

In the following theorem, we investigate the global attractivity of the equilibrium point $(0,0)$ of system (1).

Theorem 10. Assume that $\alpha \geq \beta$. Then, $(0,0)$ is a global attractor of all positive solutions of system (1).

Proof. Let $\left\{\left(x_{n}, y_{n}\right)\right\}_{n=0}^{\infty}$ be a solution of system (1). It follows from system (1) that

$$
\begin{aligned}
x_{n+1} & =(1-\alpha) x_{n}+\beta x_{n}\left(1-x_{n}\right) e^{-\left(x_{n}+y_{n}\right)} \\
& <(1-\alpha+\beta) x_{n}<x_{n}, \\
y_{n+1} & =(1-\alpha) y_{n}+\beta y_{n}\left(1-y_{n}\right) e^{-\left(x_{n}+y_{n}\right)} \\
& <(1-\alpha+\beta) y_{n}<y_{n} .
\end{aligned}
$$

Then, there exist $x \geq 0$ and $y \geq 0$ such that $\lim _{n \rightarrow \infty} x_{n}=x$ and $\lim _{n \rightarrow \infty} y_{n}=y$. Since the only possible values of $(x, y)$ in the present case are $(0,0), \lim _{n \rightarrow \infty} x_{n}=0$ and $\lim _{n \rightarrow \infty} y_{n}=$ 0 . This completes the proof.

In the following theorems, we investigate the global attractivity of the positive equilibrium point $(\bar{x} ; \bar{x})$ of system (1) where $\bar{x}$ is given by $\alpha=\beta(1-\bar{x}) e^{-2 \bar{x}}$.

Theorem 11. Assume that $\alpha+\beta e^{-2}<1$. Then the unique positive equilibrium point $(\bar{x} ; \bar{x})$ of system (1) is a global attractor of all positive solutions of system (1). 
Proof. Let $\left\{\left(x_{n}, y_{n}\right)\right\}_{n=0}^{\infty}$ be a solution of system (1), and let $x_{n} \leq \bar{x}$ (the case $x_{n} \geq \bar{x}$ is similar, and it will be left to the reader).

Now there are two cases to consider.

Case 1. Assume that $x_{0} \geq y_{0}$. Then, it follows by Theorem 1 that $x_{n}<y_{n}$ for all $n \geq 1$. Since $x_{n} \leq \bar{x}$, then $h\left(x_{n}\right) \leq 0$, where $h\left(x_{n}\right)=\alpha-\beta\left(1-x_{n}\right) e^{-2 x_{n}}$. Thus, $\alpha \leq \beta\left(1-x_{n}\right) e^{-2 x_{n}}$. Therefore, we obtain from system (1) that

$$
\begin{aligned}
x_{n+1} & =(1-\alpha) x_{n}+\beta x_{n}\left(1-x_{n}\right) e^{-\left(x_{n}+y_{n}\right)} \\
& \geq(1-\alpha) x_{n}+\alpha x_{n} e^{2 x_{n}} e^{-\left(x_{n}+y_{n}\right)} \\
& =(1-\alpha) x_{n}+\alpha x_{n} e^{x_{n}-y_{n}} \\
& \geq(1-\alpha) x_{n}+\alpha x_{n}=x_{n} .
\end{aligned}
$$

Then, the sequence $\left\{x_{n}\right\}_{n=0}^{\infty}$ is increasing, and since it was shown that it is bounded above, then it converges to the only positive equilibrium point $\bar{x}$, and it follows by the comparison test of convergence for sequence that $\left\{y_{n}\right\}_{n=0}^{\infty}$ is also convergent to the only positive equilibrium point $\bar{y}=\bar{x}$ :. Thus, $\left\{\left(x_{n}, y_{n}\right)\right\}_{n=0}^{\infty}$ converges to $(\bar{x} ; \bar{x})$.

Case 2. Assume that $x_{0}<y_{0}$. Then, it follows from system (1) and Theorem 1 that

$$
\begin{aligned}
y_{n+1} & =(1-\alpha) y_{n}+\beta y_{n}\left(1-y_{n}\right) e^{-\left(x_{n}+y_{n}\right)} \\
& \geq(1-\alpha) y_{n}+\alpha y_{n} e^{2 y_{n}} e^{-\left(x_{n}+y_{n}\right)} \\
& =(1-\alpha) y_{n}+\alpha y_{n} e^{y_{n}-x_{n}} \\
& \geq(1-\alpha) y_{n}+\alpha y_{n}=y_{n} .
\end{aligned}
$$

The rest of the proof is similar to Case 1, and it will be left to the reader.

Theorem 12. Assume that $\beta(\alpha e-\beta) \geq \alpha^{2} e^{3}$. Then, the unique positive equilibrium point $(\bar{x} ; \bar{x})$ of system (1) is a global attractor of all positive solutions of system (1).

Proof. Let $\left\{\left(x_{n}, y_{n}\right)\right\}_{n=0}^{\infty}$ be a solution of system (1). It follows from system (1) that

$$
\begin{aligned}
x_{n+1} & =(1-\alpha) x_{n}+\beta x_{n}\left(1-x_{n}\right) e^{-\left(x_{n}+y_{n}\right)} \\
& \geq(1-\alpha) x_{n}+\beta x_{n}\left(1-x_{n}\right) e^{-2} .
\end{aligned}
$$

Thus, we see from Corollary 7 that

$$
x_{n+1} \geq\left[1-\alpha+\beta\left(1-\frac{\beta}{\alpha e}\right) e^{-2}\right] x_{n} \geq x_{n} \text {. }
$$

Then, the sequence $\left\{x_{n}\right\}_{n=0}^{\infty}$ is increasing, and since it is bounded, then it converges to the only positive equilibrium point $\bar{x}$. Similarly, it is easy to show that the sequence $\left\{y_{n}\right\}_{n=0}^{\infty}$ is also convergent to the unique positive equilibrium point $\bar{y}=\bar{x}$. Therefore $\left\{\left(x_{n}, y_{n}\right)\right\}_{n=0}^{\infty}$ converges to $(\bar{x}, \bar{x})$, and then the proof is so complete.
Theorem 13. Assume that one of the following conditions hold.

(I) $5 \beta \leq 4 e^{2}(1-\alpha)$.

(II) $\alpha+\beta<1$. Then the unique positive equilibrium point $(\bar{x}, \bar{x})$ of system (1) is a global attractor of all positive solutions of system (1).

Proof. Rewrite system (1) as follows:

$$
\begin{aligned}
x_{n+1} & =F\left(x_{n}, y_{n}\right) \\
& =(1-\alpha) x_{n}+\beta\left(1-x_{n}\right) x_{n} e^{-\left(x_{n}+y_{n}\right)}, \\
y_{n+1} & =G\left(x_{n}, y_{n}\right) \\
& =(1-\alpha) y_{n}+\beta\left(1-y_{n}\right) y_{n} e^{-\left(x_{n}+y_{n}\right)}, \\
& n=0,1, \ldots,
\end{aligned}
$$

where $F(x, y)=(1-\alpha) x+\beta(1-x) x e^{-(x+y)}$ and $G(x, y)=$ $(1-\alpha) y+\beta(1-y) y e^{-(x+y)}$ are continuous functions. Now, consider the system

$$
\begin{array}{ll}
m_{1}=F\left(m_{1}, M_{2}\right), & M_{1}=F\left(M_{1}, m_{2}\right), \\
m_{2}=G\left(M_{1}, m_{2}\right), & M_{2}=F\left(m_{1}, M_{2}\right) .
\end{array}
$$

Then,

$$
\begin{aligned}
& m_{1}=(1-\alpha) m_{1}+\beta m_{1}\left(1-m_{1}\right) e^{-\left(m_{1}+M_{2}\right)}, \\
& M_{1}=(1-\alpha) M_{1}+\beta M_{1}\left(1-M_{1}\right) e^{-\left(M_{1}+m_{2}\right)}, \\
& m_{2}=(1-\alpha) m_{2}+\beta m_{2}\left(1-m_{2}\right) e^{-\left(m_{2}+M_{1}\right)}, \\
& M_{2}=(1-\alpha) M_{2}+\beta M_{2}\left(1-M_{2}\right) e^{-\left(M_{2}+m_{1}\right)} .
\end{aligned}
$$

Thus, either $m_{1}=M_{2}=m_{2}=M_{2}$ or

$$
\begin{aligned}
& \alpha=\beta\left(1-m_{1}\right) e^{-\left(m_{1}+M_{2}\right)}, \\
& \alpha=\beta\left(1-M_{1}\right) e^{-\left(M_{1}+m_{2}\right)}, \\
& \alpha=\beta\left(1-m_{2}\right) e^{-\left(m_{2}+M_{1}\right)}, \\
& \alpha=\beta\left(1-M_{2}\right) e^{-\left(M_{2}+m_{1}\right)} .
\end{aligned}
$$

Then, $m_{1}=M_{2}, m_{2}=M_{2}$ and

$$
\begin{aligned}
\left(1-m_{1}\right) e^{-2 m_{1}} & =\left(1-M_{1}\right) e^{-2 M_{1}}=\left(1-m_{2}\right) e^{-2 m_{2}} \\
& =\left(1-M_{2}\right) e^{-2 M_{2}} .
\end{aligned}
$$

Now, since $\left(1-m_{1}\right) e^{-2 m_{1}}=\left(1-M_{1}\right) e^{-2 M_{1}}$, then $e^{2\left(M_{1}-m_{1}\right)}=$ $\left(1-M_{1}\right) /\left(1-m_{1}\right)$; that is,

$$
2\left(M_{1}-m_{1}\right)=\log \left(1-M_{1}\right)-\log \left(1-m_{1}\right) .
$$

We claim that $M_{1}=m_{1}$; otherwise for the sake of contradiction assume that $M_{1}>m_{1}$ (the case where $M_{1}<m_{1}$ is similar and it will be left to the reader). Then, $\log \left(1-M_{1}\right)-$ 
$\log \left(1-m_{1}\right)<0$, which contradicts that the left hand side of (49) is positive.

Now it easy to see that

$$
\begin{gathered}
\frac{\partial F(x, y)}{\partial x}=1-\alpha+\beta\left(x^{2}-3 x+1\right) e^{-(x+y)}, \\
\frac{\partial F(x, y)}{\partial y}=-x \beta(1-x) e^{-(x+y)}, \\
\frac{\partial G(x, y)}{\partial x}=-\beta y(1-y) e^{-(x+y)}, \\
\frac{\partial G(x, y)}{\partial y}=1-\alpha+\beta\left(y^{2}-3 y+1\right) e^{-(x+y)} .
\end{gathered}
$$

Thus,

$$
\begin{aligned}
\frac{\partial F(x, y)}{\partial x} & =1-\alpha+\beta\left(x^{2}-3 x+1\right) e^{-(x+y)} \\
& \geq 1-\alpha+\beta\left(x^{2}-3 x+1\right) e^{-2} \\
& =\beta e^{-2} x^{2}-3 \beta e^{-2} x+\beta e^{-2}+1-\alpha
\end{aligned}
$$

Now, there are two cases to consider.

Case 1. Suppose that $5 \beta \leq 4 e^{2}(1-\alpha)$. Therefore, the function $w(x)=\beta e^{-2} x^{2}-3 \beta e^{-2} x+\beta e^{-2}+1-\alpha$ has no real roots. Thus, $\partial F(x, y) / \partial x \geq 0$. Similarly, it is easy to prove that $\partial G(x, y) / \partial x \geq 0$. Then, it follows by Theorem $\mathrm{A}$ that the equilibrium point $(\bar{x}, \bar{y})=(\bar{x}, \bar{x})$ of system (1) is a global attractor of all positive solutions of system (1).

Case 2. Suppose that $\alpha+2 \beta<1$. Since $0 \leq x \leq 1,3 \geq 3-x \geq$ $x(3-x)=3 x-x^{2}$, or $2 \geq 3 x-x^{2}-1$, and since $\alpha+2 \beta<$ 1 , then $1-\alpha>2 \beta>2 \beta e^{-2} \geq 2 \beta e^{-(x+y)} \geq \beta\left(3 x-x^{2}-\right.$ $1) e^{-(x+y)}$. Thus, $\partial F(x, y) / \partial x \geq 0$. Similarly, it is easy to prove that $\partial G(x, y) / \partial x \geq 0$. Then, it follows again by Theorem $\mathrm{A}$ that the equilibrium point $(\bar{x}, \bar{y})=(\bar{x}, \bar{x})$ of system (1) is a global attractor of all positive solutions of system (1). Thus, the proof is now completed.

\section{Acknowledgment}

This research was partially supported by the Deanship of Scientific Research, King Khalid University, KSA (KKU-SCI62-32).

\section{References}

[1] R. DeVault, E. A. Grove, G. Ladas, R. Levins, and C. Puccia, "Oscillation and stability in models of a perennial grass," in Proceedings of the Dynamic Systems and Applications, vol. 1, pp. 87-93, Dynamic, Atlanta, Ga, USA, 1994.

[2] R. DeVault, E. A. Grove, G. Ladas, R. Levins, and C. Puccia, "Oscillation and stability in a delay model of a perennial grass," Journal of Difference Equations and Applications, vol. 1, no. 2, pp. 173-185, 1995.

[3] E. M. ELabbasy, H. N. Agiza, H. EL-Metwally, and A. A. Elsadany, "Bifurcation analysis, chaos and control in the Burgers mapping," International Journal of Nonlinear Science, vol. 4, no. 3, pp. 171-185, 2007.

[4] E. M. Elabbasy, H. N. Agiza, A. A. Elsadany, and H. El-Metwally, "The dynamics of triopoly game with heterogeneous players," International Journal of Nonlinear Science, vol. 3, no. 2, pp. 8390, 2007.

[5] E. A. Grove, V. L. Kocic, G. Ladas, and R. Levins, "Oscillation and stability in a genotype selection model with several delays," Journal of Difference Equations and Applications, vol. 2, no. 2, pp. 205-217, 1996.

[6] E. A. Grove and G. Ladas, Periodicities in Nonlinear Difference Equations, vol. 4 of Advances in Discrete Mathematics and Applications, Chapman \& Hall/CRC, Boca Raton, Fla, USA, 2005.

[7] E. A. Grove, G. Ladas, N. R. Prokup, and R. Levins, "On the global behavior of solutions of a biological model," Communications on Applied Nonlinear Analysis, vol. 7, no. 2, pp. 33-46, 2000.

[8] V. L. Kocić and G. Ladas, Global Behavior of Nonlinear Difference Equations of Higher Order with Applications, vol. 256 of Mathematics and its Applications, Kluwer Academic Publishers Group, Dordrecht, The Netherlands, 1993.

[9] G. Papaschinopoulos, M. A. Radin, and C. J. Schinas, "On the system of two difference equations of exponential form: $x_{n+1}=$ $a+b x_{n-1} e^{y_{n}}, y_{n+1}=c+d y_{n-1} e^{x_{n}}, "$ Mathematical and Computer Modelling, vol. 54, no. 11-12, pp. 2969-2977, 2011.

[10] G. Papaschinopoulos, M. Radin, and C. J. Schinas, "Study of the asymptotic behavior of the solutions of three systems of difference equations of exponential form," Applied Mathematics and Computation, vol. 218, no. 9, pp. 5310-5318, 2012.

[11] G. Papaschinopoulos, G. Stefanidou, and K. B. Papadopoulos, "On a modification of a discrete epidemic model," Computers \& Mathematics with Applications, vol. 59, no. 11, pp. 3559-3569, 2010.

[12] D. Simsek, C. Cinar, and I. Yalcinkaya, "On the recursive sequence $x_{n+1}=\left(x_{x-3}\right) /\left(1+x_{n-1}\right)$," International Journal of Contemporary Mathematical Sciences, vol. 1, no. 9-12, pp. 475480, 2006.

[13] G. Stefanidou, G. Papaschinopoulos, and C. J. Schinas, "On a system of two exponential type difference equations," Communications on Applied Nonlinear Analysis, vol. 17, no. 2, pp. 1-13, 2010.

[14] S. Stević, "Asymptotic behaviour of a sequence de ned by iteration with applications," Colloquium Mathematicum, vol. 93, no. 2, pp. 267-276, 2002.

[15] S. Stević, "On the recursive sequence $x_{n+1}=\left(x_{x-1}\right) /\left(g\left(x_{n}\right)\right)$," Taiwanese Journal of Mathematics, vol. 6, no. 3, pp. 405-414, 2002.

[16] S. Stević, "Asymptotic behaviour of a nonlinear difference equation," Indian Journal of Pure and Applied Mathematics, vol. 34, no. 12, pp. 1681-1687, 2003.

[17] S. Stević, "On a discrete epidemic model," Discrete Dynamics in Nature and Society, vol. 2007, Article ID 87519, 10 pages, 2007.

[18] I. Yalçinkaya, C. Çinar, and M. Atalay, "On the solutions of systems of difference equations," Advances in Difference Equations, vol. 2008, Article ID 143943, 9 pages, 2008.

[19] H. A. El-Metwally and A. A. Elsadany, "Qualitative study of a bounded rationality duopoly game with exponential demand function," Utilitas Mathematica, vol. 88, pp. 337-354, 2012. 


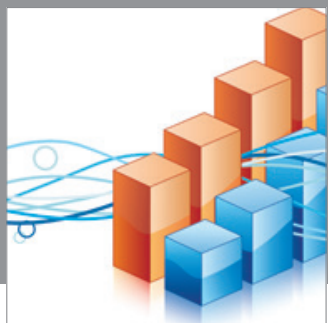

Advances in

Operations Research

mansans

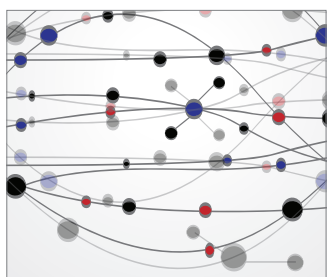

The Scientific World Journal
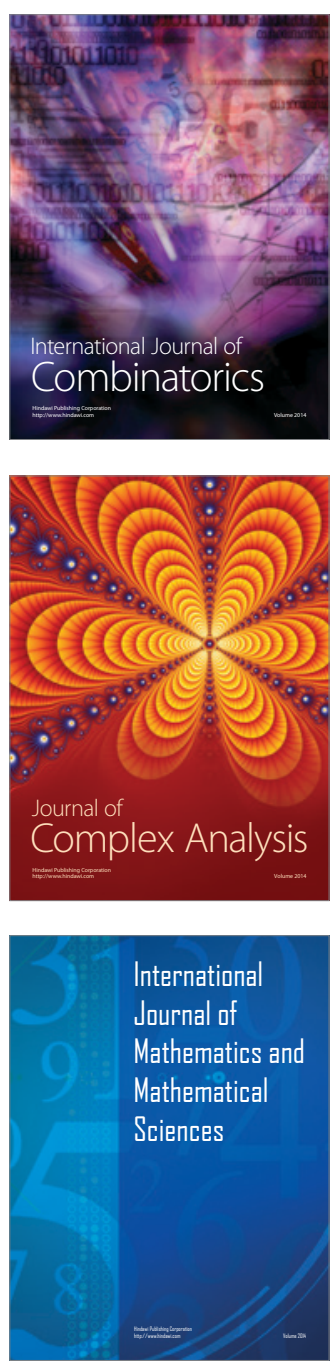
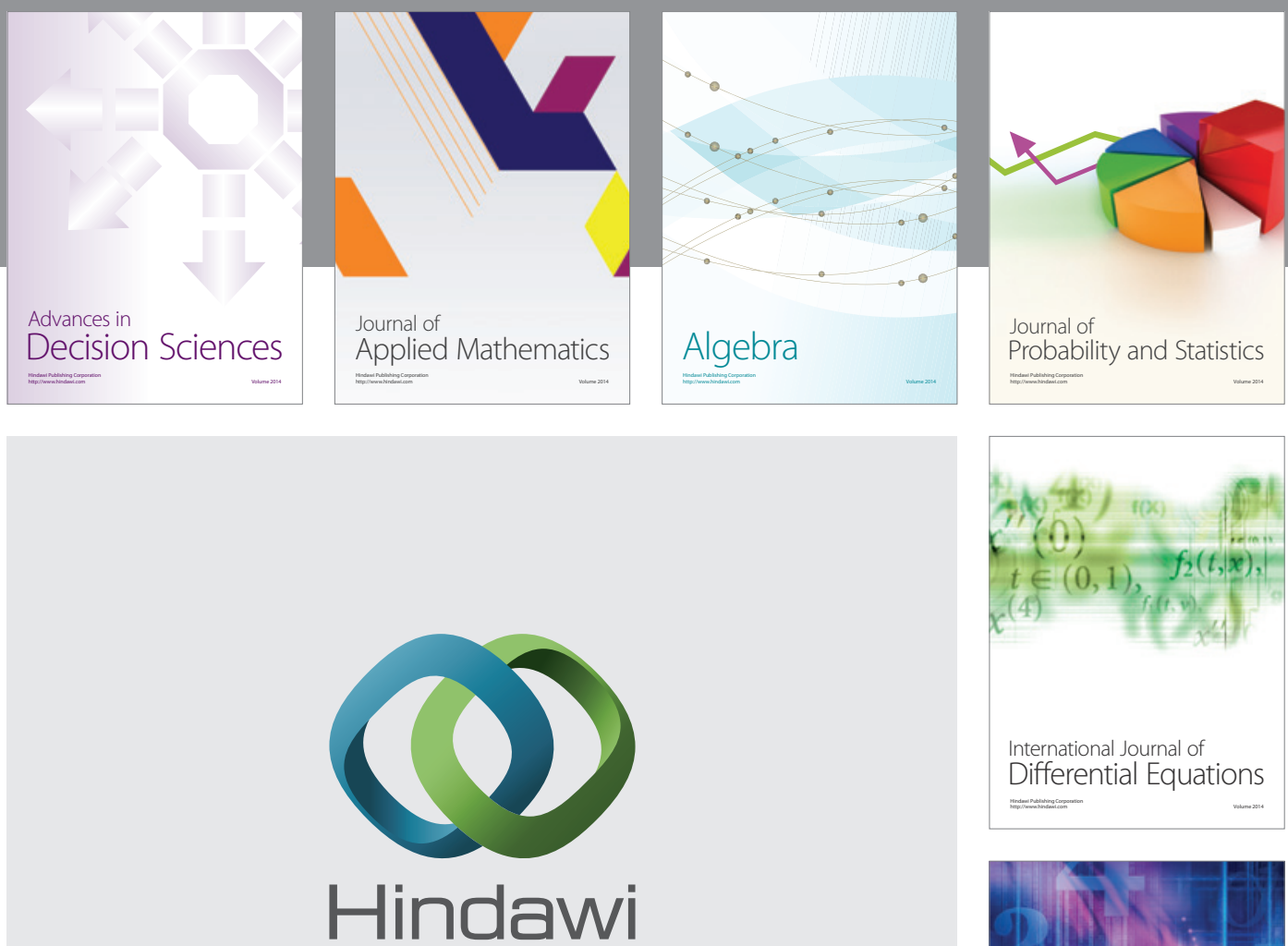

Submit your manuscripts at http://www.hindawi.com
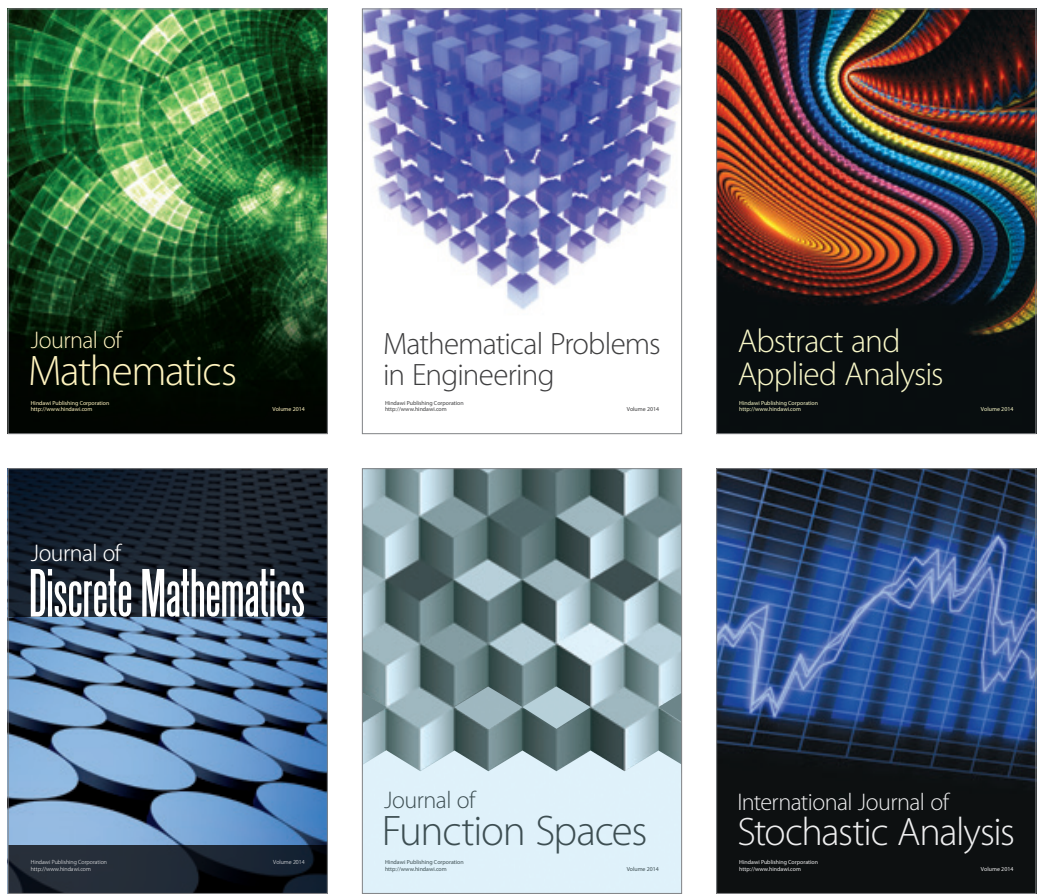

Journal of

Function Spaces

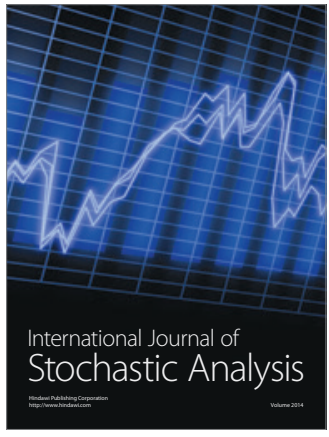

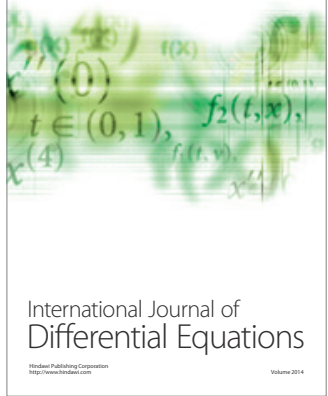
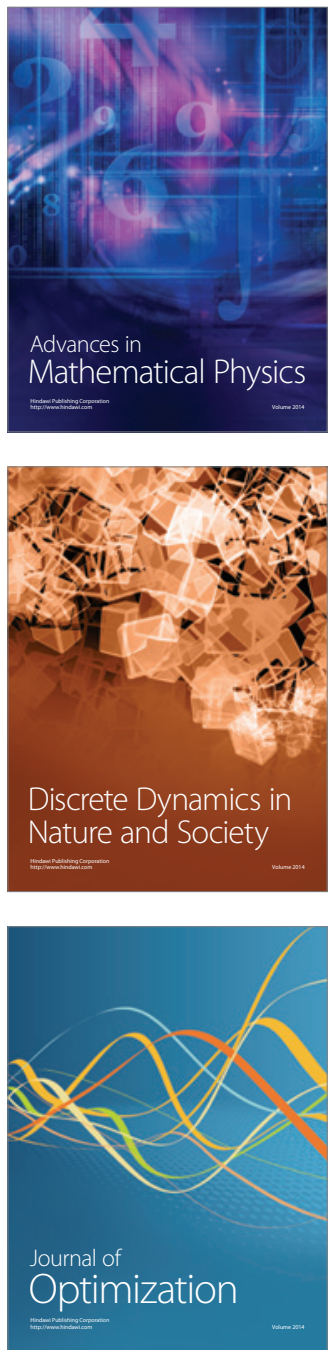